Int. J. Electrochem. Sci., 15 (2020) $1681-1690$

\title{
Preparation of an Electrochemical Luminescence Sensor for Monoamine Neurotransmitter (polydopamine) Detection after Endurance Training
}

\author{
Ying Wang
}

Department of Public Courses, Huanggang Polytechnic College, Huanggang, Hubei, 438002, P.R.

China

E-mail: supiye91j@163.com

doi: $10.20964 / 2020.02 .62$

Received: 24 August 2019 / Accepted: 26 November 2019 / Published: 31 December 2019

Electrochemiluminescence (ECL) analysis is characterized by high sensitivity and wide linear range while also providing convenient detection and simple instrumentation. Luminol, as the most commonly used luminescent reagent in chemiluminescence (CL) analysis, has been widely used in electrochemical luminescence analysis. Excitement of the dopamine (DA) system can directly promote people's curiosity and exploration, along with increasing exercise and other responses. If the DA system is inhibited, it will lead to a series of problems such as reduced movement and even a reduced desire for other life activities. In this work, a reaction of DA is catalysed by lactate dehydrogenase and coenzyme I to produce pyruvate, which reacts with pyruvate oxidase to form $\mathrm{H}_{2} \mathrm{O}_{2}$. ECL using luminol is sensitive to the above process. Based on this, a biosensor was developed to detect DA.

Keywords: Monoamine neurotransmitter; Electrochemical luminescence sensor; Endurance training; Modification; Nanomaterial

\section{FULL TEXT}

(C) 2020 The Authors. Published by ESG (www.electrochemsci.org). This article is an open access article distributed under the terms and conditions of the Creative Commons Attribution license (http://creativecommons.org/licenses/by/4.0/). 